TRANSACTIONS OF THE

AMERICAN MATHEMATICAL SOCIETY

Volume 354, Number 8, Pages 3329-3348

S 0002-9947(02)02985- 9

Article electronically published on April 3, 2002

\title{
SOLVABLE GROUPS WITH POLYNOMIAL DEHN FUNCTIONS
}

\author{
G. N. ARZHANTSEVA AND D. V. OSIN
}

\begin{abstract}
Given a finitely presented group $H$, finitely generated subgroup $B$ of $H$, and a monomorphism $\psi: B \rightarrow H$, we obtain an upper bound of the Dehn function of the corresponding HNN-extension $G=\left\langle H, t \mid t^{-1} B t=\psi(B)\right\rangle$ in terms of the Dehn function of $H$ and the distortion of $B$ in $G$. Using such a bound, we construct first examples of non-polycyclic solvable groups with polynomial Dehn functions. The constructed groups are metabelian and contain the solvable Baumslag-Solitar groups. In particular, this answers a question posed by Birget, Ol'shanskii, Rips, and Sapir.
\end{abstract}

\section{INTRODUCTION}

Recall that the Dehn function of a finitely presented group $G=\langle\mathcal{Y} \mid \mathcal{R}\rangle$ is the smallest function $\delta: \mathbb{N} \rightarrow \mathbb{N}$ with the following property. For any word $w$ over $\mathcal{Y} \cup \mathcal{Y}^{-1}$ representing the identity in $G$, there exists a van Kampen diagram over the presentation of $G$ with the number of 2 -cells at most $\delta(|w|)$, where $|w|$ denotes the length of $w$. As is well known, the asymptotic behavior of this function is essentially independent of the choice of the finite presentation of $G$. The extensive study of Dehn functions is inspired by a close connection with algorithmic problems and algebraic properties of groups. For more information and background see, for example, 19], [16], and [6].

The aim of the present paper is to investigate the Dehn functions of finitely presented HNN-extensions and construct non-polycyclic solvable groups with polynomial Dehn functions. In this section we formulate and discuss the main results of the paper and refer the reader to Section 2 for precise definitions. To formulate our results, we need an auxiliary notion. A function $f: \mathbb{N} \rightarrow \mathbb{N}$ is said to be superadditive if $f(a+b) \geq f(a)+f(b)$ for any $a, b \in \mathbb{N}$. Given an arbitrary function $f: \mathbb{N} \rightarrow \mathbb{N}$, we put

$$
\tilde{f}(n)=\max _{i=1, \ldots, n}\left(\max _{a_{1}+\cdots+a_{i}=n, a_{i} \in \mathbb{N}}\left(f\left(a_{1}\right)+\cdots+f\left(a_{i}\right)\right)\right) .
$$

In fact, $\tilde{f}$ is the smallest superadditive function such that $\tilde{f}(n) \geq f(n)$ for all $n$. For convenience, we will assume all Dehn functions under consideration to be at least linear. Besides, for any two elements $a, t$ of a group $G$, we will denote by $a^{t}$ the element $t^{-1} a t$ and by $[a, t]$ the commutator $a^{-1} t^{-1} a t$.

Received by the editors August 2, 2000 and, in revised form, October 13, 2000.

2000 Mathematics Subject Classification. Primary 20F69, 20F06, 20F65, 20F16, $20 \mathrm{~F} 05$.

Key words and phrases. Dehn function, isoperimetric function, HNN-extension, van Kampen diagram, metabelian group.

The work has been supported by the Swiss National Science Foundation. 
The following theorem plays an auxiliary role in our paper although it can be of independent interest.

Theorem 1.1. Let $H=\langle\mathcal{X} \mid \mathcal{R}\rangle$ be a finitely presented group, $B=g p\left\langle b_{1}, \ldots, b_{s}\right\rangle$ be a finitely generated subgroup of $H$ with $\left\{b_{1}, \ldots, b_{s}\right\} \in \mathcal{X}$, and $\psi: B \rightarrow H$ be a monomorphism. Let $G$ be the corresponding $H N N$-extension of $H$ with stable letter $t$, i.e.,

$$
G=\left\langle H, t \mid b_{i}^{t}=\psi\left(b_{i}\right), i=1, \ldots, s\right\rangle .
$$

Then the Dehn function of $G$ satisfies

$$
\delta_{G}(n) \preccurlyeq n \delta_{H}\left(\tilde{\Delta}_{B}^{G}(n)\right),
$$

where $\Delta_{B}^{G}(n)$ is the distortion function of $B$ in $G$.

In particular, Theorem 1.1 implies

Corollary 1.2. Under the hypothesis of Theorem 1.1, if the natural inclusion $B \rightarrow$ $G$ is a quasi-isometry, then

$$
\delta_{G}(n) \preccurlyeq n \delta_{H}(n) .
$$

Let us mention some known results in this direction. In [8], Brick investigated the Dehn functions of HNN-extensions with finite associated subgroups. Bridson and Gersten studied the Dehn function of abelian-by-cyclic groups in [9]. In the case of trivial HNN-extensions, that is

$$
G=\left\langle H, t \mid b_{i}^{t}=b_{i}, i=1, \ldots, s\right\rangle,
$$

the upper bound of the Dehn function of $G$ similar to (1.3) can be found in [11. Ch. III., , Proposition 6.20]. Moreover, in this particular case one can give a lower estimate of the Dehn function of $G$ in the following way [10]:

$$
\max \left\{\delta_{H}(n), n \Delta_{B}^{H}(n)\right\} \preccurlyeq \delta_{G}(n) .
$$

The Dehn functions of certain HNN-extensions and amalgamated products of word hyperbolic groups when associated (amalgamated) subgroups satisfy geometric conditions were studied by Kharlampovich and Myasnikov in [23] see also [4].

In a general setting the Dehn functions of amalgamated products and HNNextensions were investigated by Bernasconi in [3. However, the results from [3] are not convenient for our goal. We would like to mention that in terms of Theorem 1.1. Bernasconi showed the following estimate ([3, Corollary 5):

$$
\delta_{G}(n) \preceq n \delta_{H}\left(\lambda^{(n)}(n)\right),
$$

where $\lambda(n) \sim \max \left\{\Delta_{B}^{H}(n), \Delta_{\psi(B)}^{H}(n)\right\}+n$ and $\lambda^{(k)}(n)$ is inductively defined by $\lambda^{(1)}(n)=\lambda(n), \lambda^{(i+1)}(n)=\lambda\left(\lambda^{(i)}(n)\right)$. This estimate was obtained using the algorithm of reducing a word to a normal form in the HNN-extension and it is useful in a number of cases; on the other hand, in the best case when the embedding of $B$ in $H$ is a quasi-isometry, we obtain the exponential-type bound $\delta_{G}(n) \preceq n \delta_{H}\left(2^{n}\right)$. Thus one cannot use this result to construct groups with polynomial Dehn functions. This is the reason we need Corollary 1.2. Notice that, in contrast to the above-mentioned result of Bernasconi, Theorem 1.1 cannot be proved by using the usual algorithm for reducing a word to a normal form.

Now we are going to discuss the possibility of the lower bound for the Dehn functions of HNN-extensions. In the case of nontrivial HNN-extensions it can turn 
out that $\delta_{G}(n)$ is much smaller than $\delta_{H}(n)$. In particular, Brady [7] gave an example of a hyperbolic group $G=H \rtimes_{\psi} \mathbb{Z}$ where $H$ is finitely presented but not of type $F P_{3}$. Since any word hyperbolic group is of type $F P_{\infty}, H$ is not word hyperbolic and, in particular, $\delta_{H}(n) \succeq n^{2}$ although $\delta_{G}(n) \preccurlyeq n$ [18], [28]. Moreover, in general, it is impossible to give a lower bound for $\delta_{G}(n)$ in terms of the distortion of $B$. It will be shown in Section 3 that there is an HNN-extension presented by (1.2) such that $G$ is word hyperbolic but $\Delta_{B}^{H}(n)$ grows faster than any recursive function. However, the lower bound can be obtained in some nontrivial partial cases [17.

The following theorem shows how one can bound the Dehn function of the HNNextension given by (1.2) having no information about the distortion of $B$ in $G$ (or $B$ in $H)$.

Theorem 1.3. Let $H=\langle\mathcal{X} \mid \mathcal{R}\rangle$ be a finitely presented group, $B=g p\left\langle b_{1}, \ldots, b_{s}\right\rangle$ be a finitely generated normal subgroup of $H$, and $\psi: B \rightarrow B$ be an automorphism. Let $G$ be the corresponding HNN-extension defined by (1.2). Then the Dehn function of $G$ satisfies

$$
\delta_{G}(n) \preccurlyeq \delta_{H}\left(2^{\tilde{\delta}_{H / B}(n)}\right) .
$$

In the present paper, using Corollary 1.2, we construct first examples of finitely presented metabelian groups with polynomial Dehn functions. Note that all finitely presented solvable groups that are known to have polynomial Dehn functions are nilpotent [19, §5], [32 or polycyclic [12], 13]. The following theorem provides a source of non-polycyclic examples of solvable groups with polynomial isoperimetric functions. We recall that the Baumslag-Solitar groups are given by the presentations

$$
B S(p, q)=\left\langle a, t, \mid\left(a^{p}\right)^{t}=a^{q}\right\rangle, \quad p, q \in \mathbb{N} .
$$

It is well known that for all $q>1$ the group $B S(1, q)$ is metabelian, non-polycyclic, and has the exponential Dehn function [16].

Theorem 1.4. For any integer $q>1$, there exists a finitely presented metabelian group $G(q)$ such that the following conditions hold:

(i) $B S(1, q)$ can be quasi-isometrically embedded into $G(q)$;

(ii) $G(q)$ has at most the cubic Dehn function.

First examples of finitely presented groups $H(q)$ with the Dehn functions $\delta_{H(q)}(n)$ $\simeq n^{10}$ containing $B S(1, q)$ were obtained by Ol'shanskii and Sapir in 30. However, the groups constructed in [30] are very distant from solvable ones. To prove Theorem 1.4 we first give examples of polycyclic groups with quadratic Dehn functions using some results of Drutu 12], [13. Then we produce an HNN-extension of such a group that contains $B S(1, q)$ and use Corollary 1.2 to estimate the Dehn function of the obtained group. It is worth noticing also that the problem of finding a group $G(q)$ such as in Theorem 1.4 was suggested in [6]. This question approaches the problem of simulating of Turing machines in groups with a good control of Dehn functions using ideas of the Novikov-Boone construction [35]. We refer the interested reader to the discussion in [31, Section 2.2].

In connection with Theorem 1.4, we recall that the word problem of a group $G$ is in $N P$ (solvable in polynomial time by a non-deterministic Turing machine) if and only if $G$ can be (quasi-isometrically) embedded into a finitely presented group with polynomial Dehn function [6]. It is known that any finitely generated metabelian 
group can be represented by matrices over a finite product of fields [24, 33], 37]. It follows that the word problem is in NP for any finitely generated metabelian group. On the other hand, any finitely generated metabelian group admits an embedding into a finitely presented one [2]. Thus it is natural to ask

Question. Is any finitely generated metabelian group embeddable into a finitely presented metabelian group with polynomial Dehn function?

Theorem 1.4 provides a nontrivial reason to expect an affirmative answer to the above question.

The paper is organized as follows. In the next section we collect all necessary definitions and notation. Theorem 1.1 and Theorem 1.3 will be proved in Section 3 . Finally, the construction of groups $G(q)$ and the proof of Theorem 1.4 will be given in Section 4.

\section{Preliminary information}

2.1. Metrics and norms on groups. We begin with definitions.

Definition 2.1. Let $G$ be a group. A function dist : $G \times G \rightarrow \mathbb{R}_{+} \cup\{0\}$ is called a metric (or a distance function) if it is symmetrical, invariant under the left action of $G$ on itself, satisfies the triangle inequality, and $\operatorname{dist}(g, h)=0$ implies $g={ }_{G} h$.

Definition 2.2. We say that a non-negative function $g \rightarrow\|g\|$ on a group $G$ is a norm (or length function) if it satisfies the following conditions. For any $g, h \in G$ one has $\|g\|=\left\|g^{-1}\right\|,\|g h\| \leq\|g\|+\|h\|$, and $\|g\|=0$ implies $g=_{G} 1$.

Given a metric dist on a group $G$, one can define the corresponding norm by the formula

$$
\|g\|=\operatorname{dist}(g, 1) \text {. }
$$

Similarly, a norm on a group $G$ induces a metric by the rule

$$
\operatorname{dist}(g, h)=\left\|g^{-1} h\right\| \text {. }
$$

Recall that a map $\phi: X_{1} \rightarrow X_{2}$ between two metric spaces $\left(X_{1}\right.$, dist $\left._{X_{1}}\right)$ and $\left(X_{2}\right.$, dist $\left._{X_{2}}\right)$ is said to be a quasi-isometry if there are constants $c, \epsilon \geq 0$ and $\lambda>0$ such that the following conditions hold.

1) For any $x_{2} \in X_{2}$, there is an element $x_{1} \in X_{1}$ such that $\operatorname{dist}_{X_{2}}\left(\phi\left(x_{1}\right), x_{2}\right) \leq c$.

2) For any two elements $u, v \in X_{1}$

$$
\frac{1}{\lambda} \operatorname{dist}_{X_{1}}(u, v)-\epsilon \leq \operatorname{dist}_{X_{2}}(\phi(u), \phi(v)) \leq \lambda d i s t_{X_{1}}(u, v)+\epsilon .
$$

The metric spaces $X_{1}$ and $X_{2}$ are said to be quasi-isometric in this case.

Let us consider some important examples.

Example 2.3 (Word metrics on finitely generated groups). If a group $G$ is generated by a finite set $\mathcal{Y}$, then we define a norm on $G$ by assigning to each element $g \in G$ the length of the shortest word over $\mathcal{Y} \cup \mathcal{Y}^{-1}$ that represents $g$. The associated metric is called the word metric on $G$ corresponding to $\mathcal{Y}$. As is well known, if we take another finite generating set of $G$, we get a quasi-isometric metric space.

Example 2.4 (Riemannian metrics on Lie groups). For any connected Lie group $G$ there exists a Riemannian structure on $G$ which is invariant under the left action of $G$ on itself. This structure induces a metric dist on $G$ by the usual way. It 
is easy to see that such a metric is independent of the choice of the left invariant Riemannian structure up to quasi-isometry.

2.2. Distortions of subgroups. Let $G, H$ be groups with given norms $\|\cdot\|_{G}$, $\|\cdot\|_{H}$ and $H \leq G$. Then one defines the distortion function of $H$ in $G$ by putting

$$
\Delta_{H}^{G}(r)=\sup _{\|h\|_{G} \leq r}\|h\|_{H}
$$

for all $r \in \mathbb{R}_{+}$(see [14, 19, §3]). Clearly, if we choose other norms on $G$ and $H$ which are quasi-isometric to the original ones, we get an equivalent distortion function in the following sense. Given two functions $f, g: \mathbb{N} \rightarrow \mathbb{N}$, we put $f \preceq g$ if there exist constants $c, k>0$ such that $f(n) \leq c g(k n)$. Further, we say that $f$ is $\sim$ equivalent to $g$ and write $f \sim g$ if $f \preceq g$ and $g \preceq f$. In particular, if $G$ is a connected Lie group (respectively finitely generated group) and $H$ is a closed connected subgroup (respectively finitely generated subgroup) of $G$, then $\Delta_{H}^{G}(n)$ is well defined and independent of the choice of Riemannian (respectively word) metrics on $G$ and $H$ up to an equivalence relation. We refer to [19, §3] and [14 for more details and background.

2.3. Isoperimetric and isodiametric functions. Let $F=F(\mathcal{Y})$ be a group freely generated by $\mathcal{Y}$ and

$$
G=\langle\mathcal{Y} \mid \mathcal{R}\rangle
$$

be a finitely presented group. A function $f: \mathbb{N} \rightarrow \mathbb{N}$ is called an isoperimetric function of (2.3) if for any word $w$ in the generators $\mathcal{Y}^{ \pm 1}$ representing the identity in $G$ (i.e., $w={ }_{G} 1$ ), there is an expression

$$
w={ }_{F} \prod_{i=1}^{N} z_{i} r_{i}^{ \pm 1} z_{i}^{-1},
$$

where $r_{i} \in \mathcal{R}, z_{i} \in F$ for $i=1, \ldots, N$, and $N \leq f(|w|)$. The smallest isoperimetric function is called a Dehn function of the presentation. We denote it by $\delta_{G}(n)$.

Further, a function $h: \mathbb{N} \rightarrow \mathbb{N}$ is called an isodiametric function of the presentation (2.3) if for any word $w$ in the generators $\mathcal{Y}^{ \pm 1}, w={ }_{G} 1$, there is an expression of type (2.4) such that $\left\|z_{i}\right\|_{F} \leq h(|w|)$ for all $i=1, \ldots, N$.

The Dehn functions (respectively the smallest isodiametric functions) of any two finite presentations of the same group are equivalent in the following sense [15]. Given two functions $f, g: \mathbb{N} \rightarrow \mathbb{N}$, one defines $f \preccurlyeq g$ if there are positive constants $a, b, c, d$ such that $f(n) \leq a g(b n+c)+d n$ for all $n \in \mathcal{N}$; if $f \preccurlyeq g$ and $g \preccurlyeq f$, then one says that $f$ and $g$ are $\simeq$ equivalent. Note that in the case of an at least linear function this equivalence relation coincides with $\sim$ equivalence introduced above.

Recall that a van Kampen diagram $\Delta$ over the presentation (2.3) is an oriented planar 2-complex endowed with a label function $\varphi: E(\Delta) \rightarrow \mathcal{Y}$, where $E(\Delta)$ is the set of 1-cells (edges) of $\Delta$, such that the boundary of every 2-cell is labelled by a cyclic permutation of a word $r \in \mathcal{R}^{ \pm 1}$. For a combinatorial path $p$ in the 1-skeleton of $\Delta$ its label $\varphi(p)$ is the product of its edge labels (passing along an edge $e \in E(\Delta)$ according to its orientation we read $\varphi(e)$, otherwise we read $\left.(\varphi(e))^{-1}\right)$. By van Kampen's lemma, for a word $w$ over $\mathcal{Y} \cup \mathcal{Y}^{-1}$, one has $w={ }_{G} 1$ if and only if there is a van Kampen diagram $\Delta$ over the presentation (2.3) with the boundary label $\varphi(\partial \Delta) \equiv w$. The reader can find more details and background in [25] Ch. 5] and [29, Ch. 4]. 
The minimal number of 2-cells in $\Delta$ among all van Kampen diagrams with the boundary label $w$ is called an area of $w$ and denoted by Area $(w)$. It is easy to check that a function $f: \mathbb{N} \rightarrow \mathbb{N}$ is an isoperimetric function of (2.3) if and only if for any word $w$ over $\mathcal{Y} \cup \mathcal{Y}^{-1}, w=_{G} 1$, one has $\operatorname{Area}(w) \leq f(|w|)$.

We will also use the isoperimetric functions of Riemannian manifolds. Such a function estimates the area of an infimal surface spanning a null-homotopic loop in terms of the length of the loop. We refer to [19, §5] for a more general definition of isoperimetric functions of arbitrary metric spaces. Recall also that if a group $G$ acts properly discontinuously and cocompactly on a Riemannian manifold $M$, then $G$ is finitely generated and quasi-isometric to $M$ with respect to the word metric [36, [27. In particular, this implies that the Dehn function of $G$ is equivalent to the smallest isoperimetric function of the manifold $M[1]$.

\section{ISOPERIMETRIC FUNCTIONS OF HNN-EXTENSIONS}

3.1. The structure of van Kampen diagrams over HNN-extensions. In this section we prove Theorem 1.1 using the language of van Kampen diagrams. We will need some technical notions. The first one is so-called t-corridors (other people call them bands or strips). This notion goes back to the paper [26]; here we describe it shortly, all details can be found in [9] as well as in [6]. Suppose that one has a finite presentation of the form $\mathcal{P}=\langle\mathcal{X}, t \mid \mathcal{R}\rangle$, where $t \notin \mathcal{X}$ and the only relators involving $t$ are of the form

$$
t^{-1} x_{i} t y_{i}
$$

where $x_{i}, y_{i}$ are some words over $\mathcal{X} \cup \mathcal{X}^{-1}$. If the boundary label $\partial \pi$ of a cell $\pi$ of a van Kampen diagram over $\mathcal{P}$ is of the form (3.1), we call $\pi$ a $t$-cell. The subpath of $\partial \pi$ having the label $x_{i}$ (respectively $y_{i}$ ) is called a top (respectively a bottom) of $\pi$. A $t$-corridor is a sequence of pairwise distinct $t$-cells $\pi_{1}, \ldots, \pi_{n}$ in a van Kampen diagram over $\mathcal{P}$ such that each two consecutive cells in this sequence have a common edge labelled by $t$. The path formed by tops (respectively bottoms) of $\pi_{1}, \ldots, \pi_{n}$ is called a top (respectively a bottom) of the $t$-corridor. The length of a $t$-corridor is the number of its 2-cells. A $t$-corridor $\pi_{1}, \ldots, \pi_{n}$ is maximal if it is not contained in another $t$-corridor of greater length. Finally, a maximal $t$-corridor $\pi_{1}, \ldots, \pi_{n}$ is called a $t$-annulus if $\pi_{1}$ has no common edge with $\partial \Delta$ labelled by $t$.

We will use the following obvious information about $t$-corridors.

(1) Distinct maximal $t$-corridors have no common 2-cells.

(2) The labels of the top and the bottom of any $t$-corridor contain no $t^{ \pm 1}$.

Furthermore, $\partial \Delta$ and maximal $t$-corridors bound a number of domains that are maximal connected disjoint subcomplexes of $\Delta$ containing no edges labelled by $t$. A maximal $t$-corridor is said to be adjacent to a domain $D$ if its bottom (or its top) lies in the boundary of $D$ (as usual, we define the boundary of $D$ as the set of all external edges of $D$; an edge is called external if it belongs to the boundary of exactly one 2-cell of $D$ ). Similarly, a domain $D^{\prime}$ is called an adjacent domain to $D$ if $D$ and $D^{\prime}$ have a common adjacent $t$-corridor.

Definition 3.1. Let $\mathcal{D}$ denote the set of all domains in $\Delta$. A partial enumeration of domains in $\Delta$ is an injective map $\nu:\{1, \ldots, k\} \rightarrow \mathcal{D}$, where $k \leq$ card $\mathcal{D}$. The partial enumeration is complete if $k=\operatorname{card} \mathcal{D}$. Given a partial enumeration $\nu$, we denote $\nu(i)$ by $D_{i}$. 


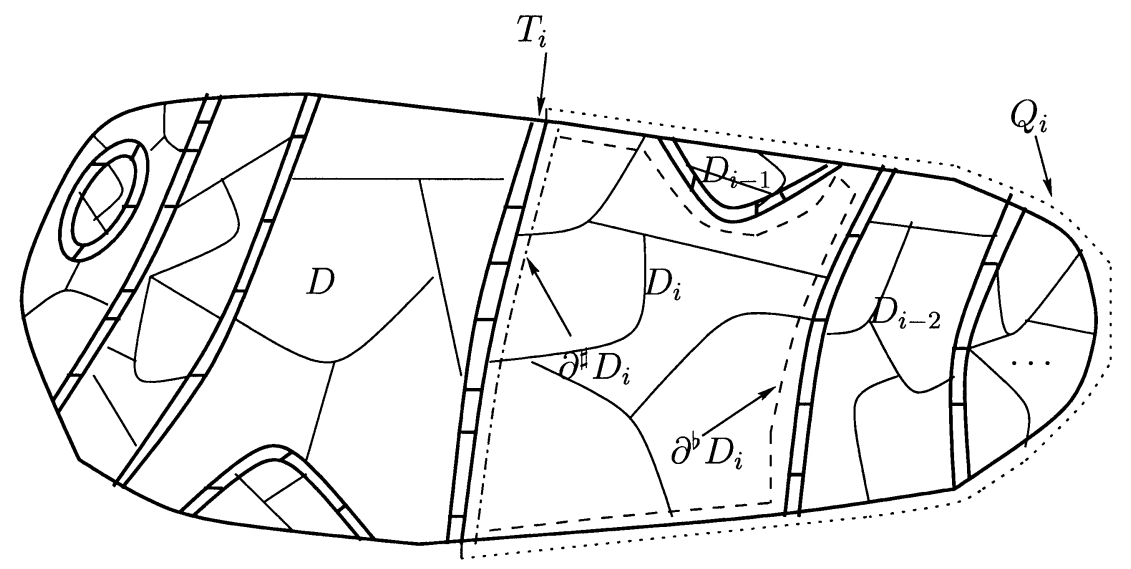

FIGURE 1.

Definition 3.2. A partial enumeration $\nu:\{1, \ldots, k\} \rightarrow \mathcal{D}$ is called regular if for any $i \in\{1, \ldots, k\}$, the domain $D_{i}$ has at most one adjacent domain from the set $\mathcal{D} \backslash\left\{D_{j}, j \leq i\right\}$.

Given a regular partial enumeration of domains in $\Delta$, assume that there is $D \in$ $\mathcal{D} \backslash\left\{D_{j}, j \leq i\right\}$ that is an adjacent domain to $D_{i} \in \mathcal{D}$. Then the unique common adjacent $t$-corridor of $D_{i}$ and $D$ is called an exterior corridor of $D_{i}$. We denote it by $T_{i}$. Thus we decompose the boundary of any $D_{i}$ into two parts

$$
\partial D_{i}=\partial^{\sharp} D_{i} \cup \partial^{b} D_{i},
$$

where $\partial^{\sharp} D_{i}$ is the top (or the bottom) of the exterior corridor $T_{i}$ of $D_{i}$ and $\partial^{\mathrm{b}} D_{i}$ is the remaining part of $\partial D_{i}$. Note that $\partial^{\sharp} D_{i}$ can be empty if the exterior corridor of $D_{i}$ does not exist.

Further, the exterior corridor $T_{i}$ of $D_{i}$ decomposes $\Delta$ into two connected subcomplexes in the natural way. We denote by $\Delta_{i}^{-}$the subcomplex containing $D_{i}$ and by $\Delta_{i}^{+}$another one. So $\Delta=\Delta_{i}^{-} \cup \Delta_{i}^{+} \cup T_{i}$ and the sets of 2-cells of $\Delta_{i}^{-}, \Delta_{i}^{+}$, and $T_{i}$ are disjoint. The part $Q_{i}=\partial \Delta_{i}^{-} \cap \partial \Delta$ of the boundary of $\Delta$ is called the assigned part for $T_{i}$. Figure 1 illustrates the introduced notion.

Lemma 3.3. Let $\nu:\{1, \ldots, k\} \rightarrow \mathcal{D}$ be a regular partial enumeration. Fix arbitrary $i \in\{1, \ldots, k\}$ and denote by $D_{j_{1}}, \ldots, D_{j_{l}}$ the adjacent domains of $D_{i}$ with numbers less then $i$, by $T_{j_{1}}, \ldots, T_{j_{l}}$ their exterior corridors, and by $Q_{j_{1}}, \ldots, Q_{j_{l}}$ the assigned parts of these corridors. Then $Q_{j_{\alpha}} \cap Q_{j_{\beta}}=\emptyset$ whenever $\alpha \neq \beta$.

Proof. Suppose that $Q_{j_{\alpha}} \cap Q_{j_{\beta}}$ is not empty. Consider the corresponding $t$-corridors $T_{j_{\alpha}}$ and $T_{j_{\beta}}$. Recall that two distinct maximal $t$-corridors have no common cells. Therefore either $\Delta_{j_{\alpha}}^{-} \subset \Delta_{j_{\beta}}^{-}$or $\Delta_{j_{\beta}}^{-} \subset \Delta_{j_{\alpha}}^{-}$. If $\Delta_{j_{\alpha}}^{-} \subset \Delta_{j_{\beta}}^{-}$, then $D_{j_{\alpha}}$ is not an adjacent domain for $D_{i}$ as $D_{i} \subset \Delta_{j_{\beta}}^{+}$. The case $\Delta_{j_{\beta}}^{-} \subset \Delta_{j_{\alpha}}^{-}$leads to a contradiction in a similar way.

3.2. Lengths of maximal $t$-corridors. Recall that the group $G$ from Theorem [1.1] is generated by $\mathcal{Y}=\mathcal{X} \cup\{t\}$. Let $w$ be a reduced word over the alphabet $\mathcal{Y} \cup \mathcal{Y}^{-1}$ that represents the identity in $G$. The following two facts are easy to check. 
(a) The numbers of occurrences of $t$ and $t^{-1}$ in $w$ are equal; we denote it by $k(w)$.

(b) If $\Delta$ is a van Kampen diagram over (1.2), $\varphi(\partial \Delta) \equiv w$, and $\Delta$ contains no $t$-annuli, then the number of domains in $\Delta$ is exactly $k(w)+1$.

Lemma 3.4. Let $G$ be the group given by (1.2) and let $w$ be a reduced word over $\mathcal{Y} \cup \mathcal{Y}^{-1}$ that represents the identity in $G$. Then for any $l \in\{0,1, \ldots, k(w)+1\}$ there exists a van Kampen diagram $\Delta$ over (1.2) with $\varphi(\partial \Delta) \equiv w$ and a regular partial enumeration $\nu:\{1, \ldots, l\} \rightarrow \mathcal{D}$ such that

(i) $\Delta$ contains no t-annuli;

(ii) for any $i \in \mathbb{N}, i \leq l$, one has

$$
\left|T_{i}\right| \leq \Delta_{B}^{G}\left(\left|Q_{i}\right|\right)
$$

where $\left|T_{i}\right|$ and $\left|Q_{i}\right|$ denote the length of $T_{i}$ and the length of the assigned part for $T_{i}$ in $\partial \Delta$ respectively.

Proof. We proceed by induction on $l$. The case $l=0$ is obvious. It means that there are no enumerated domains at all. So to satisfy the above conditions, we need to prove that there is a diagram $\Delta$ over (1.2) with boundary label $w$ without $t$-annuli. Let us take some $\Delta$ over (1.2), $\varphi(\partial \Delta) \equiv w$, and assume that $\Delta$ contains a $t$-annulus. Recall that the natural map $H \rightarrow G$ is an embedding [25. Ch. IV, Theorem 2.1]. Thus if $v$ is a word over $\mathcal{X} \cup \mathcal{X}^{-1}, v={ }_{G} 1$, then $v={ }_{H} 1$. Since the labels of the top and the bottom of any $t$-annulus contain no $t^{ \pm 1}$, we can remove the $t$-annulus together with the domain bounded by this $t$-annulus and then fill the obtained hole by cells corresponding to relators of $H$. Proceeding in this way, we can eliminate all $t$-annuli in $\Delta$ and get what we need.

Assume now that the lemma is true for $0 \leq l \leq k(w)$. Suppose $\Xi$ is a diagram without $t$-annuli over (1.2), $\mathcal{D}(\Xi)$ is the set of domains in $\Xi$, and $\mu:\{1, \ldots, l\} \rightarrow$ $\mathcal{D}(\Xi)$ is a regular partial enumeration of domains in $\Xi$ satisfying the condition (ii) above. Consider the subcomplex $\Xi_{l}^{+}$(if $l=0$ we pose $\Xi_{l}^{+}=\Xi$ ). Suppose $u \equiv \varphi\left(\partial \Xi_{l}^{+}\right)$; then $u$ equals 1 in $G$. Let $\Xi^{\prime}$ be an oriented labelled planar 2-complex obtained from $\Xi$ by removing all interior edges and vertices of $\Xi_{l}^{+}$(i.e., all edges and vertices that are not in $\partial \Xi_{l}^{+}$) and all cells of $\Xi_{l}^{+}$. By Britton's lemma [25, Ch. IV], exactly one of the assertions is true:

a) $u$ has a subword $t^{-1} b t$ with $b \in B$ and $b$ contains no $t^{ \pm 1}$;

b) $u$ has a subword $t b t^{-1}$ with $b \in \psi(B)$ and $c$ contains no $t^{ \pm 1}$;

c) $u$ contains no $t^{ \pm 1}$ at all.

Let us consider all cases.

A. Assume that the label of $\partial \Xi_{l}^{+}$is $u \equiv u_{1} t^{-1} b t u_{2}$ (when we read it starting at a suitable point), $b \in B$, and $b$ contains no $t^{ \pm 1}$ (see Figure2).

We mark by $O_{1}$ the end of the path on $\partial \Xi_{l}^{+}$labelled $u_{1} t^{-1}$ and by $O_{2}$ the end of the path on $\partial \Xi_{l}^{+}$labelled $u_{1} t^{-1} b$. Let $p$ be a path on $\partial \Delta$ started at $O_{1}$ and ended at $\mathrm{O}_{2}$ with clockwise orientation; thus $\varphi(p)={ }_{G} b$. Denote by $z \equiv b_{i_{1}}^{\varepsilon_{1}} \ldots b_{i_{m}}^{\varepsilon_{m}}$, $\varepsilon_{j}= \pm 1$, some shortest word over the alphabet $\left\{b_{1}^{ \pm 1}, \ldots, b_{s}^{ \pm 1}\right\}$, representing the same element as $\varphi(p)$ in $G$. Obviously we have

$$
m=|z| \leq \Delta_{B}^{G}(|p|)
$$

where $|p|$ denotes the combinatorial length of $p$.

Now we are going to add a new $t$-corridor to $\Xi^{\prime}$ as follows. We draw a simple combinatorial path connecting $O_{1}$ and $O_{2}$ without intersections with edges of $\Xi^{\prime}$ 


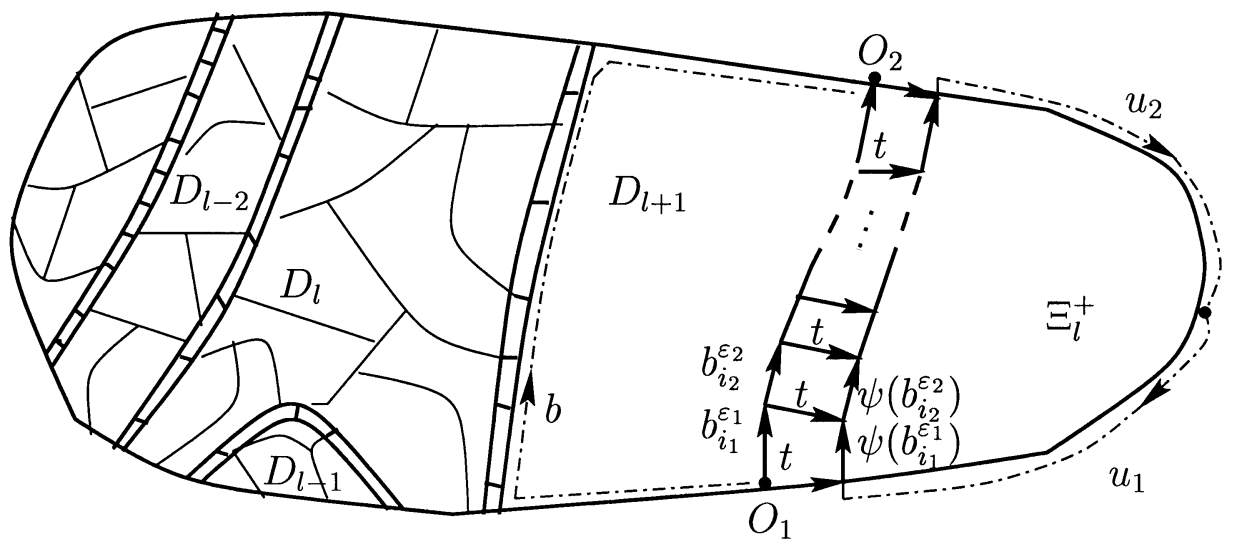

FiguRE 2.

(except for endpoints) and label it by $z$. It will be the top of the new $t$-corridor. Now the new $t$-corridor can be constructed by the natural (and unique up to a homeomorphism) way. Temporarily we call it an added $t$-corridor. The combinatorial cycle formed by the top of the added $t$-corridor and by the part of $\partial \Xi_{l}^{+}$labelled by $b$ has the label $b z^{-1}={ }_{H} \varphi(p) z^{-1}={ }_{H} 1$. So we can fill the interior of this cycle by 2-cells corresponding to relators of $H$. Similarly, since the label of the combinatorial cycle formed by the bottom of the added $t$-corridor and by the part of $\partial \Xi_{l}^{+}$labelled by $u_{2} u_{1}$ represents the identity in $G$, we can fill the interior of this cycle by 2-cells corresponding to relators of $G$ and get a van Kampen diagram $\Delta$ over (1.2) again. Moreover, taking into account the above-mentioned procedure of the elimination of $t$-annuli, we can assume without loss of generality that $\Delta$ contains no $t$-annuli.

Let $\mathcal{D}$ be the set of all domains of $\Delta$. Observe that all enumerated domains $D_{1}, \ldots, D_{l}$ of $\Xi$ have the natural isomorphic images in $\Delta$ and these images are domains too. So we can define a partial enumeration $\nu:\{1, \ldots, l+1\} \rightarrow \mathcal{D}$ as follows. If $j \in\{1, \ldots, l\}$, then $\nu(j)$ is the domain in $\Delta$ corresponding to $D_{j}=\mu(j)$. As $\nu(l+1)$ we take the subcomplex of $\Delta$ bounded by the top of the added $t$-corridor and by the part of $\partial \Xi_{l}^{+}$labelled by $b$. Since this subcomplex contains no $t$-cells, the domain $D_{l+1}$ is well-defined. Using the inductive hypothesis, it is easy to check that the constructed partial enumeration is regular. We notice that the added $t$-corridor becomes the exterior corridor to $D_{l+1}$. It remains to check that $\left|T_{i}\right| \leq \Delta_{B}^{G}\left(\left|Q_{i}\right|\right)$. Since $Q_{l+1}$ is the same as $p$, the needed inequalities follow immediately from (3.2) and the inductive hypothesis.

B. Assume that the label of $\partial \Xi_{l}^{+}$is $u=u_{1} t b t^{-1} u_{2}$ with $b \in B$ and $b$ contains no $t^{ \pm 1}$

This case is very similar to the previous one. The difference is only that $O_{1}$ is the endpoint of the path on $\partial \Xi_{l}^{+}$labelled $u_{1} t, O_{2}$ is the endpoint of the path on $\partial \Xi_{l}^{+}$ labelled $u_{1} t b$ and $\varphi(p)={ }_{G} b \in t^{-1} B t$. Hence $t \varphi(p) t^{-1}$ represents an element of $B$. Let $z \equiv b_{i_{1}}^{\varepsilon_{1}} \ldots b_{i_{m}}^{\varepsilon_{m}}$ be a shortest word over the alphabet $\left\{b_{1}^{ \pm 1}, \ldots, b_{s}^{ \pm 1}\right\}$ representing the same element as $t \varphi(p) t^{-1}$ in $G$. Then we add a new $t$-corridor whose bottom is a combinatorial path connecting $O_{1}$ and $O_{2}$ without intersections with edges of $\Xi^{\prime}$ and the top is labelled by $z$. The rest of the construction and the checking of needed properties are exactly the same as in the previous case. 
C. The label of $\partial \Xi_{l}^{+}$contains no $t^{ \pm 1}$.

In this case we fill the interior of $\partial \Xi_{l}^{+}$by 2-cells, corresponding to relators of $H$ and take the subcomplex obtained from $\Xi_{l}^{+}$as $D_{l+1}$. We do not need to check anything in this case.

Thus the inductive step is concluded in all cases. The lemma is proved.

3.3. Proof of Theorem 1.1 We leave the proof of the following trivial lemma to the reader.

Lemma 3.5. Suppose $f: \mathbb{N} \rightarrow \mathbb{N}$ is an arbitrary function and $\tilde{f}: \mathbb{N} \rightarrow \mathbb{N}$ is given by (1.1). Then the following assertions are true:

(i) the function $\tilde{f}(n)$ is superadditive, i.e., $\tilde{f}(a+b) \geq \tilde{f}(a)+\tilde{f}(b)$;

(ii) $\tilde{f}(n) \geq \max \{n, f(n)\}$ for any $n \in \mathbb{N}$;

(iii) if $f$ superadditive, then $\tilde{f} \equiv f$;

(iv) if $f(n) \leq g(n)$ for all $n \in \mathbb{N}$ and $g(n)$ is superadditive, then $\tilde{f}(n) \leq g(n)$ for all $n \in \mathbb{N}$.

Proof of Theorem 1.1. Let $w$ be a word of length $n$ that represents the identity in $G$. Using Lemma 3.4 for $l=k(w)+1$, we get a van Kampen diagram $\Delta$ without $t$-annuli with $\varphi(\partial \Delta) \equiv w$ and a complete regular enumeration $\nu:\{1, \ldots, k(w)+1\} \rightarrow \mathcal{D}$ such that for any $i \in \mathbb{N}, i \leq k(w)+1$, one has

$$
\left|T_{i}\right| \leq \Delta_{B}^{G}\left(\left|Q_{i}\right|\right) \leq \Delta_{B}^{G}(n) .
$$

Let us remove all interior edges, vertices and all 2-cells of each domain $D \in \mathcal{D}$. Since $\varphi(\partial D)$ contains no $t^{ \pm 1}$ for any $D \in \mathcal{D}$, we can obtain a van Kampen diagram over (1.2) by filling the interior of $\partial D$ by at most $\delta_{H}(|\partial D|)$ cells corresponding to relators of $H$ for each $D \in \mathcal{D}$.

Let us estimate $\left|\partial D_{i}\right|$ for a fixed $D_{i} \in \mathcal{D}$. We put $M=\max _{i=1, \ldots, s}\left|\psi\left(b_{i}\right)\right|$. Let $D_{j_{1}}, \ldots, D_{j_{m}}$ be the adjacent domains of $D_{i}$ such that $j_{\alpha}<i$ for all $\alpha=1, \ldots, m$, $T_{j_{1}}, \ldots, T_{j_{m}}$ be their exterior corridors, and $Q_{j_{1}}, \ldots, Q_{j_{m}}$ the assigned parts of these corridors.

By Lemma 3.3 we have $Q_{j_{\alpha}} \cap Q_{j_{\beta}}=\emptyset$ whenever $\alpha \neq \beta$. Hence

$$
\sum_{\alpha=1}^{m}\left|Q_{j_{\alpha}}\right| \leq n=|w|
$$

Using (3.3), (3.4), and Lemma 3.5, we obtain

$$
\left|\partial^{b} D_{i}\right| \leq M \sum_{\alpha=1}^{m}\left|T_{j_{\alpha}}\right|+|\partial \Delta| \leq M \sum_{\alpha=1}^{m} \Delta_{B}^{G}\left(\left|Q_{j_{\alpha}}\right|\right)+n \leq M \tilde{\Delta}_{B}^{G}(n)+n
$$

and

$$
\left|\partial^{\sharp} D_{i}\right| \leq M\left|T_{i}\right| \leq M \Delta_{B}^{G}(n) .
$$

This and Lemma 3.5 imply

$$
\left|\partial D_{i}\right|=\left|\partial^{\mathrm{b}} D_{i}\right|+\left|\partial^{\sharp} D_{i}\right| \leq 2 M \tilde{\Delta}_{B}^{G}(n)+n \leq(2 M+1) \tilde{\Delta}_{B}^{G}(n) .
$$


Clearly, we have the same estimate for all domains of $\Delta$. As we supposed that any Dehn function is at least linear, we have

$$
\begin{aligned}
& \operatorname{Area}(w) \leq(k(w)+1) \delta_{H}\left((2 M+1) \tilde{\Delta}_{B}^{G}(n)\right)+\sum_{i=1}^{k(w)}\left|T_{i}\right| \\
& \leq n \delta_{H}\left((2 M+1) \tilde{\Delta}_{B}^{G}(n)\right)+n \Delta_{B}^{G}(n) \preccurlyeq n \delta_{H}\left(\tilde{\Delta}_{B}^{G}(n)\right) .
\end{aligned}
$$

3.4. Distortions of normal subgroups in finitely generated groups. Let $L$ be a finitely generated group and $N$ be a normal finitely generated subgroup of $L$. Observe that in general, the distortion of $N$ in $L$ can be arbitrarily large. To show this we provide here an elegant argument suggested by Sela [19, §3, 3K"]. Recall that if $L$ is a finitely presented group with solvable word problem and $N$ is a finitely generated normal subgroup of $L$, then the word problem in $L / N$ is solvable if and only if the distortion of $N$ in $L$ is bounded by some recursive function [14. It follows from Rips construction [34], that for any finitely presented group $Q$, there exists a short exact sequence

$$
1 \longrightarrow N \longrightarrow L \longrightarrow Q \longrightarrow 1
$$

where $L$ is word hyperbolic and $N$ is finitely generated. Now assume that $Q$ is a finitely presented group with unsolvable word problem. Then the distortion function of $N$ in $L$ grows faster than any recursive function (in particular, than any iterated exponent).

However, we can estimate the distortion of a normal subgroup using the Dehn function of the quotient group.

Lemma 3.6. Let $L$ be a finitely presented group, and $N$ a finitely generated normal subgroup of $L$. Then we have

$$
\Delta_{N}^{L}(n) \preccurlyeq 2^{\delta_{L / N}(n)} .
$$

Proof. Suppose $L$ is given by a finite presentation $L=\langle\mathcal{X} \mid \mathcal{R}\rangle$. Let $\mathcal{S}$ be a finite generating set of $N$. Then $L / N$ has a presentation

$$
\langle\mathcal{X} \mid \mathcal{S} \cup \mathcal{R}\rangle \text {. }
$$

Take an element $h \in N$ of length $\|h\|_{L}=n$ with respect to $\mathcal{X}$. Let $w$ be a shortest word over $\mathcal{X} \cup \mathcal{X}^{-1}$ representing $h$. Then $w$ can be written (in the free group $F(\mathcal{X})$ ) as

$$
w={ }_{F(\mathcal{X})} \prod_{l=1}^{p} g_{l}^{-1} s_{i_{l}}^{ \pm 1} g_{l} \cdot \prod_{l=1}^{q} f_{l}^{-1} r_{j_{l}}^{ \pm 1} f_{l}
$$

where $s_{i_{l}} \in \mathcal{S}, r_{j_{l}} \in \mathcal{R}, g_{l}, f_{l} \in F(\mathcal{X})$, and $p+q=\delta_{L / N}(n)$. In particular, we have

$$
p \leq \delta_{L / N}(n) .
$$

Note that if $f_{L / N}(n)$ is the isodiametric function of $L / N$, then $f_{L / N}(n) \preccurlyeq \delta_{L / N}(n)$ (the proof of this fact for any finitely presented group is just a trivial exercise; as an alternative, the reader can find the proof in [15]). In particular, this means that 
there is a constant $c>0$ which is independent of $h$ such that $\left\|g_{l}\right\|_{F(\mathcal{X})} \leq c \delta_{L / N}(n)$ for all $l=1, \ldots, p$. Set $M=\max _{s \in \mathcal{S}, x \in \mathcal{X}_{\cup} \cup \mathcal{X}^{-1}}\left\|x^{-1} s x\right\|_{N}$. Then

$$
\left\|g_{l}^{-1} s_{i_{l}}^{ \pm 1} g_{l}\right\|_{N} \leq M^{\left\|g_{l}\right\|_{F(\mathcal{X})}} \leq M^{c \delta_{L / N}(n)} .
$$

Combining (3.6) and (3.7), we obtain

$$
\|h\|_{N}=\left\|\prod_{l=1}^{p} g_{l}^{-1} s_{i_{l}}^{ \pm 1} g_{l}\right\|_{N} \leq \sum_{l=1}^{p}\left\|g_{l}^{-1} s_{i_{l}}^{ \pm 1} g_{l}\right\|_{N} \leq \delta_{L / N}(n) M^{c \delta_{L / N}(n)} \preccurlyeq 2^{\delta_{L / N}(n)} .
$$

This implies (3.5).

3.5. Proof of Theorem 1.3. To prove the theorem we need the following simple observation. Let $U, V$ be finitely presented groups. Then the Dehn function of the free product $W=U * V$ satisfies

$$
\delta_{W}(n) \leq \max \left\{\tilde{\delta}_{U}(n), \tilde{\delta}_{V}(n)\right\} .
$$

The proof is straightforward and is left to the reader.

Proof of Theorem 1.3. Under the hypothesis of Theorem 1.3. notice that $B$ is a normal subgroup in $G$ and $G / B \cong(H / B) *\langle t\rangle$. Therefore $\Delta_{B}^{G}(n) \preceq 2^{\delta_{(H / B) *\langle t\rangle}(n)}$ in view of Lemma 3.6. By the result of Guba and Sapir [20], the Dehn function of $(H / B) *\langle t\rangle$ is superadditive. It is not hard to check that this implies the superadditivity of the function $2^{\delta_{(H / B) *\langle t\rangle}(n)}$. Using (iv) of Lemma 3.5 and (3.8), we obtain

$$
\tilde{\Delta}_{B}^{G}(n) \preccurlyeq 2^{\delta_{(H / B) *\langle t\rangle}(n)} \preccurlyeq 2^{\tilde{\delta}_{(H / B)}(n)} .
$$

To conclude the proof, it remains to refer to Theorem 1.1

3.6. One example. In a contrast to the case of trivial HNN-extensions, in general, it is impossible to give a lower bound for the Dehn function of HNN-extension given by (1.2) using only the information about the distortion of $B$ in $H$ (or $B$ in $G$ ). Indeed, let $Q$ be a finitely presented group with unsolvable word problem. Consider the free product $\langle t\rangle * Q$ and take a word hyperbolic group $L$ such that $\langle t\rangle * Q=L / N$ for some finitely generated subgroup $N$ of $L$. Such a group does exist by the abovementioned Rips construction. Clearly, $L$ splits into an HNN-extension with stable letter $t$, the associated subgroup $N$, and the base group $T$, where $T$ is the preimage of $Q$ under the natural epimorphism $L \rightarrow\langle t\rangle * Q$. It remains to note that $\Delta_{N}^{L}(n)$ and $\Delta_{N}^{T}(n)$ both grow faster that any recursive function, although $\delta_{L}(n) \simeq n$.

\section{Metabelian groups With POLynomial isoperimetric FunCtions}

4.1. Polycyclic groups with quadratic Dehn functions. The aim of the present section is to prove Theorem 1.4 First we provide examples of polycyclic groups which have quadratic Dehn functions using results of the papers [12], [13] and study certain properties of our groups needed for the sequel. 
Definition 4.1. A monomorphism $\alpha: \mathbb{R}^{n-1} \rightarrow G L(n, \mathbb{R})$ is said to be generic if the image $\alpha\left(\mathbb{R}^{n-1}\right)$ is conjugated to a torus of the form

$$
\left\{\left(\begin{array}{cccc}
e^{\lambda_{1}} & 0 & \ldots & 0 \\
0 & e^{\lambda_{2}} & \ldots & 0 \\
\vdots & \vdots & \ddots & \vdots \\
0 & 0 & \cdots & e^{\lambda_{n}}
\end{array}\right), a_{1} \lambda_{1}+\cdots+a_{n} \lambda_{n}=0\right\}
$$

with $a_{1} \cdots a_{n} \neq 0$.

The following result can be found in 13 .

Proposition 4.2. Suppose $Q=\mathbb{R}^{n} \rtimes_{\alpha} \mathbb{R}^{n-1}, n \geq 3$, where $\alpha: \mathbb{R}^{n-1} \rightarrow G L(n, \mathbb{R})$ is a generic monomorphism. Then $Q$ has a quadratic isoperimetric function.

We note that the above proposition was proved in [12] with an asymptotically cubic estimate of isoperimetric function. In the recent paper 13] the estimation was improved to a quadratic. It is worth noticing that the results of [12] and [13] can be applied to a much larger class of groups.

Lemma 4.3. Suppose that $S$ and $A$ are free abelian groups with free generating sets $\left\{s_{1}, \ldots, s_{n-1}\right\}$ and $\left\{a_{1}, \ldots, a_{n}\right\}$ respectively. Assume that $\beta: S \rightarrow S L(n, \mathbb{Z})$ is a monomorphism such that the image $\beta\left(s_{i}\right)$ is semi-simple and $\operatorname{Spec}\left(\beta\left(s_{i}\right)\right) \subset \mathbb{R}$ for all $i=1, \ldots, n-1$. Let $P=A \rtimes_{\beta} S$ be the split extension given by the presentation

$$
P=\left\langle\begin{array}{l|l}
a_{1}, \ldots, a_{n}, s_{1}, \ldots, s_{n-1} & \begin{array}{l}
{\left[a_{i}, a_{j}\right]=\left[s_{k}, s_{l}\right]=1, a_{i}^{s_{k}}=a_{1 i}^{\mu_{1 i}^{(k)}} \ldots a_{n}^{\mu_{n i}^{(k)}},} \\
i, j=1, \ldots, n ; k, l=1, \ldots, n-1
\end{array}
\end{array}\right\},
$$

where $\left(\mu_{1 i}^{(k)}, \ldots, \mu_{n i}^{(k)}\right)$ is the $i$-th column of $\beta\left(s_{k}\right)$. Then the Dehn function of $P$ is quadratic whenever $n \geq 3$.

Proof. Since the group $S$ is abelian, there is an element $\theta \in S L(n, \mathbb{R})$ such that $\theta \beta\left(s_{i}\right) \theta^{-1}=\operatorname{diag}\left(\nu_{i 1}, \ldots, \nu_{i n}\right)$ with some $\nu_{i j} \neq 0, i=1, \ldots, n-1$. Without loss of generality we can assume $\nu_{i j}>0$ (indeed, we can take a subgroup $P_{0}$ of $P$ generated by $A$ and $s_{1}^{2}, \ldots, s_{n-1}^{2}$; clearly, $P_{0}$ is of finite index in $P$, and hence the Dehn functions of $P_{0}$ and $P$ are equivalent). Let us define a homomorphism $\gamma: \mathbb{R}^{n-1} \rightarrow S L(n, \mathbb{R})$ by

$$
\gamma\left(t e_{i}\right)=\operatorname{diag}\left(e^{t \ln \nu_{i 1}}, \ldots, e^{t \ln \nu_{i n}}\right),
$$

where $e_{i}$ is the $i$-th standard basis vector in $\mathbb{R}^{n-1}, t \in \mathbb{R}$. Since $\theta \beta(S) \theta^{-1}$ is discrete in $S L(n, \mathbb{R}), \gamma\left(\mathbb{R}^{n-1}\right)$ contains a discrete subgroup isomorphic to $\mathbb{Z}^{n-1}$. Obviously this implies $\gamma\left(\mathbb{R}^{n-1}\right)=\mathbb{R}^{n-1}$. So $\gamma$ is an injection. It is also clear that $\gamma$ is generic. Consider the Lie group $Q=\mathbb{R}^{n} \rtimes_{\gamma} \mathbb{R}^{n-1}$. By Proposition 4.2, $Q$ has a quadratic Dehn function. Further, let us note that the natural inclusions $A \rightarrow \mathbb{R}^{n}$ and $S \rightarrow \mathbb{R}^{n-1}$ induce an embedding $\varkappa: P \rightarrow Q$. It can easily be shown that $\varkappa(P)$ is a uniform lattice in $Q$. This implies that $P$ is quasi-isometric to $Q$ and therefore the Dehn functions of $Q$ and $P$ are equivalent [1].

In connection with the condition $n \geq 3$ in the above lemma, we note that the Dehn function of the group of type $\mathbb{Z}^{m} \rtimes \mathbb{Z}$ is polynomial if and only if the group is virtually nilpotent; otherwise, the Dehn function is exponential [9]. 
Now we want to give an explicit construction of polycyclic groups $P$ satisfying the hypothesis of Lemma 4.3. In order to do this, we need some information from algebraic number theory.

Lemma 4.4. Let $k$ be a finite normal extension of $\mathbb{Q}$. Denote by $k_{0}$ the ring of algebraic integers of $k$ and by $k_{0}^{*}$ the group of units of $k_{0}$. Since $k_{0}$ is a free module of dimension $n=[k: \mathbb{Q}]$ over $\mathbb{Z}$, the action of $k_{0}^{*}$ on $k_{0}$ by multiplication induces a monomorphism

$$
\alpha: k_{0}^{*} \rightarrow S L(n, \mathbb{Z})
$$

Suppose that the Galois group $G(k / \mathbb{Q})$ is simple; then the image $\alpha(\xi)$ is semi-simple for any $\xi \in k_{0}^{*}$.

Proof. Assume that $\alpha(\xi)$ is not semi-simple for some $\xi \in k_{0}^{*}$. Then the characteristic polynomial $\chi(x)$ of $\alpha(\xi)$ has multiple roots. Therefore, $\chi(x)$ splits into two nontrivial factors as follows:

$$
\chi(x)=v(x)\left(\chi(x), \chi^{\prime}(x)\right)
$$

where $\chi^{\prime}(x)$ is the derivative of $\chi(x)$ and $\left(\chi(x), \chi^{\prime}(x)\right)$ denotes the greatest common divisor of $\chi(x)$ and $\chi^{\prime}(x)$. Clearly, $v(x) \in \mathbb{Q}[x]$ and $\operatorname{deg} v(x)<n$. Moreover, any root of $\chi(x)$ is a root of $v(x)$. Since $\xi$ acts on $k_{0}$ by multiplication, $\chi(\xi)=0$. It follows that $v(\xi)=0$. Recall that $k$ is normal. This implies that the splitting field $l$ of $v(x)$ is a subfield of $k$. Moreover, $l$ is a nontrivial normal extension of $\mathbb{Q}$ and

$$
[l: \mathbb{Q}]=\operatorname{deg} v(x)<\operatorname{deg} \chi(x)=n .
$$

Thus $\mathbb{Q} \neq l \neq k$ that contradicts the simplicity of the Galois group.

Now let us take $k$, a purely real normal extension of $\mathbb{Q}$ of degree $n \geq 3$, such that the Galois group $G(k / \mathbb{Q})$ is simple. As an exercise, the reader can check that the splitting field of the polynomial $x^{3}-3 x+1$ satisfies these conditions; in fact, $G(k / \mathbb{Q}) \cong \mathbb{Z} / 3 \mathbb{Z}$ in this case. Since $k$ is purely real, $k_{0}^{*}$ is a finite extension of $\mathbb{Z}^{n-1}$ by Dirichlet's Theorem (see [22, Appendix] or [21, III. 28]). Hence the restriction of $\alpha$ to the subgroup of $k_{0}^{*}$ isomorphic to $\mathbb{Z}^{n-1}$ gives an embedding of $\mathbb{Z}^{n-1}$ into $S L(n, \mathbb{Z})$ satisfying the assumptions of Lemma 4.3. Indeed, by Lemma 4.4 $\alpha(\xi)$ is semi-simple for all $\xi \in k_{0}^{*}$. Since the characteristic polynomial $\chi(x)$ of any $\alpha(\xi)$, $\xi \in k_{0}^{*}$, has a root in $k, \chi(x)$ splits into linear factors over $k$. This means that $\operatorname{Spec}(\alpha(\xi)) \subset k \subset \mathbb{R}$ for all $\xi$ in $k_{0}^{*}$.

Lemma 4.5. Let $G$ be a connected Lie group, $\mathbb{X}$ be one of the additive groups $\mathbb{R}, \mathbb{Z}$, and $\phi: \mathbb{X} \rightarrow G$ be a monomorphism. Suppose that there exist $g \in G$ and $\lambda \in \mathbb{X}$, $\lambda>1$, such that $g^{-1} \phi(1) g=\phi(\lambda)$. Then the image $\phi(\mathbb{X})$ endowed with the natural metric is a strictly exponentially distorted subgroup of $G$, i.e., there are $c, \varepsilon>0$ such that

$$
\|\phi(x)\|_{G} \leq c \log _{2}(1+|x|)+\varepsilon
$$

for all $x \in \mathbb{X}$.

Proof. For any $n \in \mathbb{X}, n>1$, we have

$$
n=\epsilon_{0}+\epsilon_{1} \lambda+\ldots+\epsilon_{j} \lambda^{j}=\epsilon_{0}+\lambda\left(\epsilon_{1}+\ldots+\lambda\left(\epsilon_{j-1}+\lambda \epsilon_{j}\right) \ldots\right),
$$


where $j \leq \log _{\lambda} n$ and $\epsilon_{i} \in[0 ; \lambda)$ for all $i$. Denote by $l(k)$ the length $\|\phi(k)\|_{G}$. The equality $g^{-1} \phi(1) g=\phi(\lambda)$ implies $g^{-1} \phi(k) g=\phi(\lambda k)$ for all $k \in \mathbb{X}$. Therefore we have $l\left(k_{1}+k_{2}\right) \leq l\left(k_{1}\right)+l\left(k_{2}\right)$ and $l(\lambda k) \leq l(k)+2\|g\|_{G}$. Hence

$$
l(n) \leq l\left(\epsilon_{0}\right)+\sum_{i=1}^{j}\left(l\left(\epsilon_{i}\right)+2\|g\|_{G}\right) \leq\left(M+2\|g\|_{G}\right) \log _{\lambda} n+M,
$$

where $M=\sup _{\epsilon \in[0, \lambda)} l(\epsilon)$. The lemma is proved.

Lemma 4.6. Suppose $P$ is the group given by (4.1) and $A$ is the abelian subgroup of $P$ generated by $\left\{a_{1}, \ldots, a_{n}\right\}$. Then $A$ is strictly exponentially distorted in $P$, i.e., there are constants $c, \varepsilon>0$ such that

$$
\|a\|_{G} \leq c \log \left(1+\|a\|_{A}\right)+\varepsilon
$$

for any $a \in A$.

Proof. Recall that in the proof of Lemma 4.3 we have constructed the group $Q=$ $E \rtimes_{\gamma} D$, where $E=\mathbb{R}^{n}, D=\mathbb{R}^{n-1}$, and $\gamma$ is given by 4.2). Since $P$ can be embedded into $Q$ as a lattice such that the image of $A$ will be a lattice in $E$, to prove the lemma it is sufficient to show that $E$ is strictly exponentially distorted in $Q$.

Denote by $\mathfrak{e}$ the subalgebra of the Lie algebra $\mathfrak{q}$ of $Q$ corresponding to $E$. Let us fix a basis $X_{1}, \ldots, X_{n}$ of $\mathfrak{e}$ such that the adjoint action of $D$ on $\mathfrak{e}$ is defined by $\gamma$. By Lemma 4.5, the one-parameter subgroups corresponding to $X_{1}, \ldots, X_{n}$ are strictly exponentially distorted in $Q$. So there is a collection of positive constants $c_{1}, \ldots, c_{n}, \varepsilon_{1}, \ldots, \varepsilon_{n}$ such that

$$
\left\|\exp \left(t X_{i}\right)\right\|_{Q} \leq c_{i} \log (|t|+1)+\varepsilon_{i}
$$

for all $t \in \mathbb{R}$, and $i=1, \ldots, n$. Set $c=\max _{i=1, \ldots, n} c_{i}$ and $\varepsilon=\max _{i=1, \ldots, n} \varepsilon_{i}$. Suppose now that $f=\exp (X) \in E, X \in \mathfrak{e}$. Then

$$
X=a_{1} X_{1}+\ldots+a_{n} X_{n}
$$

for some constants $a_{1}, \ldots, a_{n} \in \mathbb{R}$. Assume that the set $\{1, \ldots, n\}$ is a disjoint union of two subsets $I_{1}$ and $I_{2}$ such that $\left|a_{i}\right|<2$ if $i \in I_{1}$, and $\left|a_{i}\right| \geq 2$ whenever $i \in I_{2}$. Set $n_{0}=\sharp I_{2}$.

Since $E$ is abelian, we can take the Euclidean metric on $E$. This means that

$$
\|f\|_{E}=\sqrt{\sum_{i=1}^{n}\left|a_{i}\right|^{2}} \geq \sqrt{\sum_{i \in I_{2}}\left|a_{i}\right|^{2}} \geq \sqrt[n_{0}]{\prod_{i \in I_{2}}\left|a_{i}\right|}
$$

On the other hand, the inequalities $|a| \geq 2$ and $|b| \geq 2$ imply $|a|+|b| \leq|a b|$ and, therefore, $(|a|+1)(|b|+1)=|a b|+|a|+|b|+1<2(|a b|+1)$. By induction, we obtain

$$
\prod_{i \in I_{2}}\left(\left|a_{i}\right|+1\right) \leq 2^{n_{0}}\left(\prod_{i \in I_{2}}\left|a_{i}\right|+1\right)
$$


Finally, using (4.3) we have

$$
\begin{aligned}
\|f\|_{Q}= & \left\|\prod_{i=1}^{n} \exp \left(a_{i} X_{i}\right)\right\|_{Q} \leq \sum_{i=1}^{n}\left\|\exp \left(a_{i} X_{i}\right)\right\|_{Q} \leq \sum_{i=1}^{n}\left(c_{i} \log \left(\left|a_{i}\right|+1\right)+\varepsilon_{i}\right) \\
& \leq \sum_{i \in I_{1}}\left(c_{i} \log \left(\left|a_{i}\right|+1\right)+\epsilon_{i}\right)+\sum_{i \in I_{2}}\left(c_{i} \log \left(\left|a_{i}\right|+1\right)+\epsilon_{i}\right) \\
& \leq\left(n-n_{0}\right)(c \log 3+\epsilon)+\sum_{i \in I_{2}}\left(c_{i} \log \left(\left|a_{i}\right|+1\right)+\epsilon_{i}\right) \\
& \leq c \log \left(2^{n_{0}}\left(\prod_{i \in I_{2}}\left|a_{i}\right|+1\right)\right)+n_{0} \epsilon+\left(n-n_{0}\right)(c \log 3+\epsilon) \\
& \leq c \log \left(\left(\|f\|_{E}\right)^{n_{0}}+1\right)+c n_{0} \log 2+n \epsilon+c\left(n-n_{0}\right) \log 3 \\
& \leq c n_{0} \log \left(\|f\|_{E}+1\right)+c n \log 3+n \epsilon .
\end{aligned}
$$

The lemma is proved.

4.2. Construction of $G(q)$. Now suppose $P$ is some group given by the presentation (4.1), where $\beta$ satisfies the assumptions of the Lemma 4.3.

Lemma 4.7. The map $\phi: P \rightarrow P$ defined by

$$
\begin{array}{ll}
a_{i} \mapsto a_{i}^{q}, & i=1, \ldots, n, \\
s_{j} \mapsto s_{j}, & j=1, \ldots, n-1
\end{array}
$$

induces an isomorphism of $P$.

Proof. It is easy to see that the images of the defining relations of $P$ under $\phi$ represent the identity in $P$. Therefore $\phi$ is an endomorphism of $P$. Next observe that if $\phi(w){ }_{P} 1$ for some nontrivial word $w$ over $\left\{a_{1}, \ldots, a_{n}, s_{1}, \ldots, s_{n-1}\right\}^{ \pm 1}$, then $w$ represents an element of $A$ (otherwise $\phi(w)$ represents a nontrivial element in the quotient group $P / A$ since $\left.\phi(w) A={ }_{P / A} w A\right)$. Thus $w=_{P} a_{1}^{\lambda_{1}} \ldots a_{n}^{\lambda_{n}}$. However, $\phi(w)=_{P} a_{1}^{q \lambda_{1}} \ldots a_{n}^{q \lambda_{n}}$ and $\phi(w)=_{P} 1$ imply $\lambda_{i}=0$ for all $i$ that contradicts to $w \neq{ }_{P} 1$.

Let $G(q)$ be the HNN-extension of $P$ associated to $\phi$, i.e.,

$$
G(q)=\left\langle P, t \mid a_{i}^{t}=a_{i}^{q},\left[s_{j}, t\right]=1, i=1, \ldots, n, j=1, \ldots, n-1\right\rangle .
$$

We denote by $\mathcal{X}$ the alphabet $\left\{a_{1}, \ldots, a_{n}, s_{1}, \ldots, s_{n-1}\right\}$ and set $\mathcal{Y}=\mathcal{X} \cup\{t\}$.

4.3. The distortion of $P$ in $G(q)$. If $w$ is a word in the alphabet $\mathcal{Y}^{ \pm 1}$, then we denote by $\alpha(w)$ the number of appearances of $a_{i}^{ \pm 1}, i=1, \ldots, n$, in $w$, by $\sigma(w)$ the number of appearances of $s_{j}^{ \pm 1}, j=1, \ldots, n-1$, in $w$, and by $\tau_{+}(w), \tau_{-}(w)$ the number of appearances of $t$ and $t^{-1}$ in $w$ respectively. We set $\tau(w)=\tau_{+}(w)+\tau_{-}(w)$.

Lemma 4.8. Let $u$ be a word in the alphabet $\mathcal{X}^{ \pm 1}$. Then there is a unique word $u^{\prime}$ representing the same element of $P$ as $u$ such that

$$
u^{\prime} \equiv \prod_{j=1}^{n-1} s_{j}^{\xi_{j}} \cdot \prod_{i=1}^{n} a_{i}^{\epsilon_{i}} .
$$


Moreover, we have

$$
\alpha\left(u^{\prime}\right) \leq \alpha(u) M^{\sigma(u)}, \quad \sigma\left(u^{\prime}\right) \leq \sigma(u),
$$

where $M=\max \left\{\left\|\beta\left(s_{j}\right)\right\|,\left\|\beta\left(s_{j}^{-1}\right)\right\| ; j=1, \ldots, n-1\right\}$ and $\left\|\beta\left(s_{j}^{ \pm 1}\right)\right\|$ denotes the norm of the corresponding linear operator.

Proof. Using the equalities

$$
\begin{aligned}
a_{i}^{ \pm 1} s_{k}= & { }_{G} s_{k} a_{1}^{ \pm \mu_{1 i}^{(k)}} a_{2}^{ \pm \mu_{2 i}^{(k)}} \ldots a_{n}^{ \pm \mu_{n i}^{(k)}} \\
a_{i}^{ \pm 1} s_{k}^{-1}= & { }_{G} s_{k}^{-1} a_{1}^{ \pm \nu_{1 i}^{(k)}} a_{2}^{ \pm \nu_{2 i}^{(k)}} \ldots a_{n}^{ \pm \nu_{n i}^{(k)}} \\
& s_{k}^{ \pm 1} s_{l}^{ \pm 1}={ }_{G} s_{l}^{ \pm 1} s_{k}^{ \pm 1} \\
& a_{i}^{ \pm 1} a_{j}^{ \pm 1}={ }_{G} a_{j}^{ \pm 1} a_{j}^{ \pm 1}
\end{aligned}
$$

for $i, j=1, \ldots, n, k, l=1, \ldots, n-1$, we can transform $u$ into a word of type (4.6) by applying a collecting process. Namely, first we take an appearance of $s_{1}^{ \pm 1}$ in $u$, and remove it from right to left using the above formulas. Then we take another appearance of $s_{1}^{ \pm 1}$ in $u$ and proceed in the same way and so on. We continue this procedure until all appearances of $s_{1}^{ \pm 1}$ will be collected and we will get a word of type $s_{1}^{\xi_{1}} v$, where $v$ contains no $s_{1}^{ \pm 1}$. Then we do the same for $s_{2}, \ldots, s_{n-1}, a_{1}, \ldots, a_{n-1}$. Clearly, we get a word of type (4.6) when the collected process has been completed. It is not hard to check that the inequalities (4.7) hold.

Now suppose that

$$
u^{\prime \prime} \equiv \prod_{j=1}^{n-1} s_{j}^{\xi_{j}^{\prime}} \cdot \prod_{i=1}^{n} a_{i}^{\epsilon_{i}^{\prime}}
$$

is another word representing the same element as $u$ in $P$. We get immediately $\xi_{j}=\xi_{j}^{\prime}$ for all $j$ considering the images of $u^{\prime}$ and $u^{\prime \prime}$ in $P / A$. This implies that

$$
\prod_{i=1}^{n} a_{i}^{\epsilon_{i}^{\prime}}={ }_{A} \prod_{i=1}^{n} a_{i}^{\epsilon_{i}} .
$$

Since $A$ is free abelian, $\epsilon_{i}=\epsilon_{i}^{\prime}$ for all $i$.

Lemma 4.9. Let $P$ and $G(q)$ be the groups given by (3.6) and (4.5) respectively. Then $P$ is undistorted in $G(q)$. This means that there exists a constant $c>0$ such that

$$
\Delta_{P}^{G(q)}(n) \leq c n
$$

for any $n \in \mathbb{N}$.

Proof. Let $p$ be an element of $P$ and $w$ be a shortest word over $\mathcal{Y}$ representing $p$. It is obvious that $\tau_{+}(w)=\tau_{-}(w)=\tau(w) / 2$. Using the equalities

$$
\begin{gathered}
a_{i}^{ \pm 1} t={ }_{G} t a_{i}^{ \pm q}, \\
t^{-1} a_{i}^{ \pm 1}={ }_{G} a_{i}^{ \pm q} t^{-1}, \\
s_{j}^{ \pm 1} t^{ \pm 1}={ }_{G} t^{ \pm 1} s_{j}^{ \pm 1},
\end{gathered}
$$


for $i=1, \ldots, n, j=1, \ldots, n-1$, we transform $w$ into

$$
w_{1} \equiv t^{\rho} u t^{-\rho}
$$

where $u$ is a word over $\mathcal{X}$ and $0 \leq \rho \leq \frac{\tau(w)}{2}$. Clearly, $\sigma\left(w_{1}\right)=\sigma(w)$ and $\alpha\left(w_{1}\right) \leq$ $\alpha(w) q^{\tau(w)}$. Further, take the word

$$
u^{\prime} \equiv \prod_{j=1}^{n-1} s_{j}^{\xi_{j}} \cdot \prod_{i=1}^{n} a_{i}^{\epsilon_{i}}
$$

representing the same element as $u$. By Lemma 4.8, we have

$$
\begin{gathered}
\sigma\left(u^{\prime}\right) \leq \sigma(u)=\sigma(w), \\
\alpha\left(u^{\prime}\right) \leq \alpha(u) M^{\sigma(u)} \leq \alpha(w) q^{\tau(w)} M^{\sigma(w)} .
\end{gathered}
$$

The uniqueness of the representative of type (4.6) implies that $\epsilon_{i}=\chi_{i} q^{\frac{\tau(w)}{2}}$, for some $\chi_{i} \in \mathbb{Z}, i=1, \ldots, n$. Therefore the word

$$
w_{2} \equiv \prod_{j=1}^{n-1} s_{j}^{\xi_{j}} \cdot \prod_{i=1}^{n} a_{i}^{\chi_{i}}
$$

represents the element $p$. By Lemma 4.6, there exist constants $c, c^{\prime}, \varepsilon$ independent of $p$ such that

$$
\begin{aligned}
\|p\|_{P} & \leq\left\|\prod_{j=1}^{n-1} s_{j}^{\xi_{j}}\right\|_{P}+\left\|\prod_{i=1}^{n} a_{i}^{\chi_{i}}\right\|_{P} \leq \sigma\left(w_{2}\right)+c \log _{2}\left(1+\sum_{i=1}^{n}\left|\chi_{i}\right|\right)+\varepsilon \\
& \leq \sigma(w)+c \log _{2}\left(1+\alpha(w) q^{\tau(w)} M^{\sigma(w)}\right)+\varepsilon \leq c^{\prime}|w|+\varepsilon=c^{\prime}\|p\|_{G}+\varepsilon .
\end{aligned}
$$

The lemma is proved.

4.4. Proof of Theorem 1.4. To show that the Baumslag-Solitar group $B S(1, q)$ can be embedded into $G(q)$ we need the following simple lemma.

Lemma 4.10. Let $B S(1, q)$ be the Baumslag-Solitar group given by 1.7), $K$ a group, and $\eta: B S(1, q) \rightarrow K$ a homomorphism. Suppose that $\eta(a)$ is of infinite order in $K$; then $\eta$ is a monomorphism.

Proof. Let us take a nontrivial element $w$ of $B S(1, q)$. Suppose that $\eta(w)={ }_{K} 1$. If $w$ lies in the normal closure $\langle a\rangle^{B S(1, q)}$ of the cyclic subgroup $\langle a\rangle$ in $B S(1, q)$, then $w^{t^{k}}={ }_{B S(1, q)} a^{m}$ for some $k, m \in \mathbb{Z}$. Thus $(\eta(a))^{m}={ }_{K} 1$ that contradicts to the assumption of the lemma. Further, suppose $w \notin\langle a\rangle^{B S(1, q)}$. Then $w \in t^{l}\langle a\rangle^{B S(1, q)}$ for some $l \neq 0$. Hence

$$
\eta(a)={ }_{K} \eta(a)^{\eta(w)}={ }_{K} \eta\left(a^{t^{l}}\right)={ }_{K}(\eta(a))^{q^{l}} .
$$

Thus $(\eta(a))^{q^{l}-1}={ }_{K} 1$, and we get a contradiction again.

Proof of Theorem 1.4 Since the natural map $P \rightarrow G(q)$ is an embedding [25, Ch. 4, Theorem 2.1], the element $a_{1}$ is of infinite order in $G(q)$. In view of Lemma4.10 this implies that the subgroup of $G(q)$ generated by $a_{1}$ and $t$ is isomorphic to $B S(1, q)$.

Let us show that the embedding of $B S(1, q)$ into $G(q)$ is quasi-isometric. The proof of this fact is very similar to that of Lemma 4.9] so we omit details and give a sketch of the proof only. Suppose $b$ is an element of $B S(1, q)$ and $w$ is 
some shortest word over $\left\{a_{1}, \ldots, a_{n}, s_{1}, \ldots, s_{n-1}, t\right\}^{ \pm 1}$ representing $b$. Using the collecting process as in the proof of the Lemmas 4.8 and 4.9, it is not hard to show that $w$ can be transformed into a word $w^{\prime}$ of type $t^{\rho} a_{1}^{\varepsilon} t^{-\rho}$, where

$$
|\rho| \leq \frac{\tau(w)}{2}, \quad \text { and } \varepsilon \leq \alpha(w)(\max \{q, M\})^{\sigma(w)+\tau(w)} .
$$

By Lemma 4.5, the cyclic subgroup $\left\langle a_{1}\right\rangle$ is strictly exponentially distorted in $\left\langle a_{1}, t\right\rangle$. Thus we have

$$
\begin{aligned}
\|b\|_{\left\langle a_{1}, t\right\rangle}=\left\|t^{\rho} a_{1}^{\varepsilon} t^{-\rho}\right\|_{\left\langle a_{1}, t\right\rangle} & \leq \tau(w)+c \log \left(1+\alpha(w)(\max \{q, M\})^{\sigma(w)+\tau(w)}\right)+\varepsilon \\
& \leq c^{\prime}|w|+\varepsilon=c^{\prime}\|b\|_{G(q)}+\varepsilon
\end{aligned}
$$

for some constants $c, c^{\prime}, \varepsilon$ independent of $b$.

It remains to prove that $G(q)$ has at most a cubic Dehn function. We know already that $P$ has a quadratic Dehn function. By Lemma 4.9 $P$ is an undistorted subgroup of $G(q)$. Using Corollary 1.2 we get immediately that $\delta_{G}(n) \preccurlyeq n^{3}$.

4.5. Final remarks. Probably it is not hard to extend Theorem 1.4 and get an embedding of any torsion free finitely presented abelian-by-cyclic group into a finitely presented metabelian group with cubic Dehn function. The reason is that any torsion free finitely presented abelian-by-cyclic group has a presentation which is similar (in some sense) to the canonical presentations of Baumslag-Solitar groups [5].

\section{ACKNOWLEDGMENTS}

We are grateful to M. Sapir for useful discussions and explanations concerning the construction of polycyclic groups with quadratic Dehn functions. We also thank the referee who directed our attention to A. Bernasconi's work.

\section{REFERENCES}

1. J. M. Alonso, Inégalités isopérimétriques et quasi-isométries, C.R. Acad. Sci. Paris Série 1 311 (1990), 761-764. MR 91k:57004

2. G. Baumslag, On finitely presented metabelian groups, Bull. Amer. Math. Soc. 78 (1972), 279. MR 45:354

3. Aldo A. Bernasconi, On HNN-extensions and the complexity of the word problem for one-relator groups, Ph.D. thesis, University of Utah, June 1994; available on http://www.math.utah.edu/ gersten/Papers/bernasconi-thesis.ps.gz.

4. M. Bestvina and M. Feighn, A combination theorem for negatively curved groups, J. Differential Geom. 35(1) (1992), 85-101. MR 93d:53053

5. R. Bieri and R. Strebel, Almost finitely presented soluble groups, Comment. Math. Helv. 53 (1978), 258-278. MR 58:16890

6. J. C. Birget, A. Yu. Ol'shanskii, E. Rips, and M. V. Sapir, Isoperimetric functions of groups and computational complexity of the word problem, (1998), preprint.

7. N. Brady, Branched coverings of cubical complexes and subgroups of hyperbolic groups, J. London Math. Soc. 60(2) (1999), 461-480. MR 2000j:20076

8. S. G. Brick, On Dehn functions and products of groups, Trans. Amer. Math. Soc. 335 (1993), 369-384. MR 93c:57003

9. M. R. Bridson and S.M. Gersten, The optimal isoperimetric inequality for torus bundles over the circle, Quart. J. Math. 47 (1996), 1-23. MR 97c:20047

10. M.R. Bridson, Fractional isoperimetric inequalities and subgroup distortion, J. Amer. Math. Soc. 12(4) (1999), 1103-1118. MR 2001a:20062

11. M. R. Bridson and A. Haefliger, Metric spaces of non-positive curvature, Grundlehren der Mathematischen Wissenschaften [Fundamental Principles of Mathematical Sciences], 319, Springer-Verlag, Berlin, 1999. MR 2000k:53038 
12. C. Drutu, Remplissage dans des réseaux de Q-rang 1 et dans des groupes résolubles, Pacific J. Math. 185(2) (1998), 269-305. MR 99m:22009

13. C. Drutu, Filling in lattices and solvable groups, (2000), preprint.

14. B. Farb, The extrinsic geometry of subgroups and the generalized word problem, Proc. London Math. Soc. 68(3) (1994), 577-593. MR 94m:20073

15. S.M. Gersten, Isoperimetric and isodiametric functions of finite presentations, Geometric Group Theory (G. Niblo, R. Roller, ed.), vol. 1, LMS Lecture Note Series 181, Cambridge Univ. Press, 1993, 79-96. MR 94f:20066

16. S.M. Gersten, Dehn functions and $l_{1}$-norms of finite presentations, Algorithms and classification in combinatorial group theory (Berkeley, CA, 1989), Math. Sci. Res. Inst. Publ. 23, Springer, New York, 1992, 195-224. MR 94g:20049

17. S.M. Gersten, Some remarks on subgroup of hyperbolic groups, (1999), preprint.

18. M. Gromov, Hyperbolic groups, Essays in group theory (S.M. Gersten, ed.), MSRI series, vol. 8, Springer-Verlag, 1987. MR 89e:20070

19. M. Gromov, Asymptotic invariants of infinite groups, Geometric group theory, 2 (Sussex, 1991), 1-295, London Math. Soc. Lecture Note Ser., 182, Cambridge Univ. Press, Cambridge, 1993. MR 95m:20041

20. V.S. Guba, M.V. Sapir, On Dehn functions of free products of groups, Proc. Amer. Math. Soc. 127(7) (1999), 1885-1891. MR 99j:20044

21. H. Hasse, Number theory, Springer-Verlag, 1980. MR 81c:12001b

22. M. I. Kargapolov, Ju. I. Merzljakov, Fundamentals of the theory of groups, Springer, 1979. MR 80k:20002

23. O. Kharlampovich, A. Myasnikov, Hyperbolic groups and free constructions, Trans. Amer. Math. Soc. 350 (1998), no. 2, 571-613. MR 98d:20041

24. E. M. Levich, The representation of two-step solvable groups by matrices over a field of characteristic zero, Mat. Sb. 81 (1970), 352-357.

25. R. Lyndon and P. Schupp, Combinatorial group theory, Springer-Verlag, 1977. MR 58:28182

26. Ch.F. Miller III, P.E. Schupp, The geometry of Higman-Neumann-Neumann extensions, Comm. Pure and Appl. Math. XXVI (1973), 787-802. MR 49:9091

27. J. Milnor, A note on curvature and the fundamental group, J. Diff. Geom. 2 (1968), 1-7. MR 38:636

28. A. Yu. Ol'shanskii, Hyperbolicity of groups with subquadratic isoperimetric inequality, Int. J. Algebra Comput. 1(3) (1991), 281-289. MR 93d:20067

29. A. Yu. Ol'shanskii, The geometry of defining relations in groups, Kluwer Academic Publishers, 1991. MR 93g:20071

30. A. Yu. Ol'shanskii and M. V. Sapir, Embeddings of relatively free groups into finitely presented groups, Combinatorial and computational algebra (Hong Kong, 1999), 23-47, Contemp. Math., 264, Amer. Math. Soc., Providence, RI, 2000. MR 2001j:20043

31. A. Yu. Ol'shanskii, and M. V. Sapir, Length and area functions on groups and quasi-isometric Higman embeddings, Internat. J. Algebra Comput. 11(2) (2001), 137-170. MR 2002b:20058

32. C. Pittet, Isoperimetric inequalities in nilpotent groups, J. London Math. Soc. 55(3) (1997), 588-600. MR 98h:20060

33. V. N. Remeslennikov, Representation of finitely generated metabelian groups by matrices, Algebra i Logika, 8 (1969), 72-75 (Russian). MR 44:335

34. E. Rips, Subgroups of small cancellation groups, Bull. London Math. Soc. 14 (1982), 45-47. MR 83c:20049

35. J. Rotman, An introduction to the theory of groups, Allyn \& Bacon, 3rd edition, 1984. MR 85f:20001

36. A.S. Švarc, Volume invariants of coverings, Dokl. Akad. Nauk 105 (1955), 32-34 (Russian). MR 17:781d

37. B. A. F. Wehrfritz, On finitely generated soluble linear groups, Math. Z. 170(2) (1980), 155-167. MR 81f:20063

Section de Mathématiques, Université de Genève, CP 240, 1211 Genève 24, SwitzerLAND

E-mail address: Goulnara.Arjantseva@math.unige.ch

Department of High Algebra, mehmat, Moscow State University, 119899 Moscow, RUSSIA

E-mail address: Denis.Osin@mtu-net.ru 